\title{
Eficiência in vitro de três diluidores para sêmen de coelho
}

André Furugen Cesar de ANDRADE $^{1}$

Eneiva Carla Carvalho

CELEGHINI ${ }^{1}$

Letícia Andresa

YONEZAWA $^{1}$

Aleksandrs SPERS ${ }^{2}$

Rubens Paes de ARRUDA ${ }^{1}$

\section{Correspondência para :}

Prof. Rubens Arruda, (19) 3565-4221/35654262, Av. Duque de Caxias Norte, 225, Caixa Postal 23, Pirassununga - SP, 13630-000,arrudarp@usp.br

Recebido para publicação: 20/02/2006 Aprovado para publicação: 30/10/2008

\author{
1 - Centro de Biotecnologia em Reprodução Animal do Departamento de \\ Reprodução Animal da Faculdade de Medicina Veterinária e Zootecnia da \\ Universidade de São Paulo, Pirassununga-SP \\ 2 - Departamento de Nutrição e Produção Animal da Faculdade de Medicina \\ Veterinária e Zootecnia da Universidade de São Paulo, Pirassununga-SP
}

\section{Resumo}

Foi o objetivo deste trabalho comparar in vitro a eficiência de três diluidores para sêmen de coelhos: solução de Ringer com lactato de sódio, um meio à base de citrato de sódio e gema de ovo e um meio à base de leite desnatado sobre a manutenção da motilidade progressiva e do vigor espermático. Para tanto, foram utilizados 5 coelhos realizando-se 10 colheitas de sêmen de cada $(n=50)$. O sêmen foi colhido com vagina artificial, e avaliado quanto ao volume, motilidade, vigor e concentração. O sêmen foi diluído (20x106 espermatozóides/ $\mathrm{mL}$ ) em microtubos pré-aquecidos a $37^{\circ} \mathrm{C}$ nos três diluidores, e então incubados em banho-maria a $37^{\circ} \mathrm{C}$ por 120 minutos, realizando-se avaliações a cada 30 minutos. Imediatamente após a diluição (tempo $0)$ a motilidade espermática não diferiu $(\mathrm{P}>0,05)$ entre os diluidores, todavia, reduziu $(\mathrm{P}<0,05)$ no diluído com Ringer quando comparado ao sêmen in natura. Já o vigor no tempo 0 diminuiu $(\mathrm{P}<0,05)$ nos três meios. A motilidade espermática foi melhor preservada $(\mathrm{P}<0,05)$ durante a incubação de 30 até 120 minutos para o sêmen diluído nos diluidores citrato-gema e à base de leite do que para aquele diluído em Ringer. A preservação do vigor variou entre os diluidores durante o tempo de incubação in vitro, contudo, foi semelhante entre os três diluidores após 120 minutos de incubação. Com estes resultados, pode-se inferir que os diluidores testados proporcionam um meio que permite manter a viabilidade espermática para que possa ser realizada a inseminação artificial em coelhas até duas horas pós-diluição.

\section{Introdução}

A técnica da inseminação artificial (IA) em diversas espécies constituiu uma ferramenta básica para o progresso zootécnico registrado nas últimas décadas. Em coelhos, a IA vem sendo usada na produção intensiva de coelhos como uma ferramenta para a seleção genética, aumento da fertilidade em épocas estacionais desfavoráveis e controle sanitário. ${ }^{1,2} \mathrm{~A}$ técnica possibilitou o desenvolvimento de um novo sistema de produção, chamado de sistema de produção cíclica, que consiste em agrupar animais no mesmo estágio fisiológico da esfera reprodutiva, permitindo inseminar as coelhas em dias fixos. ${ }^{3}$

$\mathrm{Na}$ Europa a IA vem sendo empregada em larga escala, como uma tecnologia para aumentar a eficiência da indústria cunícola. Todavia, no Brasil a monta natural vem sendo utilizada como o principal método para reprodução em coelhos. A introdução da IA na prática rotineira da produção poderia trazer grandes avanços para o desenvolvimento da cunicultura nacional, que vem apresentando acentuada evolução ao longo desta década, nos aspectos da tecnificação da produção, com melhorias na conformação da carcaça e aumento da produção de alimento de qualidade ao consumidor. ${ }^{4}$ 
A diluição do sêmen é crucial para o emprego da IA, pois permite melhor aproveitamento de cada ejaculado e conservação do sêmen por um período de tempo maior.

A dose inseminante para coelhos preconizada é em torno de 10 milhões de espermatozóides viáveis ${ }^{5}$, podendo varia de 8 a 11,1 milhões ${ }^{6}$ em um volume inseminante de $0,5 \mathrm{~mL} .{ }^{5,6}$ Se levarmos em consideração que o volume ejaculado de um coelho varia de 0,5 a $2,0 \mathrm{~mL}^{6}$ e a média da concentração espermática para um coelho de porte médio varia de $250^{7}$ a 500 milhões $/ \mathrm{mL}^{8}$; tem-se ejaculados de 125 milhões até 1 bilhão de espermatozóides, ou seja, cada ejaculado é suficiente para a IA de mais de 12 fêmeas.

A propriedade dos diluidores em conservar o sêmen se deve a sua composição, que varia das mais simples até mais elaboradas, dependendo do objetivo da diluição, seja para servir como um simples extensor e proporcionar maior volume ou para a conservação por um período de tempo maior, resfriado ou congelado. Assim, em geral sua constituição deve garantir nutrição, proteção, balanço eletrolítico ( $\mathrm{pH}$ e osmolaridade) e inibição bacteriana. ${ }^{9}$

Para a IA de coelhos, a diluição do sêmen pode ser realizada empregando-se extensores simples como solução fisiológica ou solução de Ringer com lactato de sódio ${ }^{10,11,12}$ ou um diluidor mais específico como o citrato-gema modificado, com apenas $5 \%$ de gema de ovo $^{5}$, além de diluidores à base de leite desnatado ${ }^{13}$. Todavia, poucos estudos foram conduzidos com o objetivo de comparar os diluidores para o sêmen de coelhos.

Dessa forma, o presente estudo teve o propósito de comparar in vitro a eficiência da solução de Ringer com lactato de sódio, um meio à base de leite desnatado e um meio à base de gema de ovo e citrato de sódio, como diluidores para sêmen de coelhos sobre a manutenção da motilidade progressiva e do vigor espermático, incubados à temperatura de $37^{\circ} \mathrm{C}$ por um período de até 120 minutos.

\section{Material e Método}

Para este estudo foram utilizados cinco animais, realizando-se 10 colheitas de sêmen de cada animal $(\mathrm{n}=50)$. O sêmen foi colhido com vagina artificial, modelo descrito por Andrade et al. ${ }^{14}$, com o auxílio de um manequim vivo. Após a colheita do sêmen, a fração gelatinosa foi separada e o sêmen foi avaliado quanto ao volume, motilidade, vigor e concentração. As amostras de sêmen foram imediatamente diluídas em uma concentração de 20 milhões de espermatozóides $/ \mathrm{mL}$ em microtubos pré-aquecidos a $37^{\circ} \mathrm{C}$, nos seguintes diluidores: solução de Ringer com lactato de sódio (J.P. Indústria Farmacêutica, Ribeirão Preto/Brasil) (R), meio citrato-gema $5 \%{ }^{5}$ (C - tabela 1), ou meio à base de leite desnatado $^{15}(\mathrm{~L}$ - tabela 1$)$. Após a diluição o sêmen foi incubado em banho-maria a $37^{\circ} \mathrm{C}$ por 120 minutos, sendo realizadas cinco avaliações de motilidade e vigor de cada diluidor nos tempos 0 (imediatamente após a diluição), 30, 60, 90 e 120 minutos de incubação in vitro.

A motilidade e o vigor foram determinados pela deposição de uma gota de sêmen entre lâmina e lamínula préaquecidas a $37^{\circ} \mathrm{C}$, sendo a motilidade determinada pela porcentagem de espermatozóides com movimento progressivo e o vigor avaliado com base na qualidade do movimento retilíneoprogressivo e sua velocidade em uma escala de 1 a 5 , sendo ambas as avaliações realizadas sob microscopia óptica de contraste de fase em aumento de 100x. A concentração espermática foi determinada pela contagem das células em câmara de Neubauer, após diluição da amostra de sêmen em formol salino tamponado $(1: 100)$, com uso de microscopia óptica sob aumento de 400x.

$\mathrm{O}$ experimento foi delineado em blocos generalizados sendo cada coelho um 
Tabela 1 - Fórmula do diluidor citrato-gema 5\% (C) e do meio à base de leite desnatado (L) ${ }^{15}$ - Pirassununga, 2004

\begin{tabular}{ccc}
\hline Produto & Componente & Quantidade \\
\hline & Citrato de sódio & $1,47 \mathrm{~g}$ \\
Citrato-gema 5\% (C) & Penicilina G Potássica & $5,056 \mathrm{~g}$ \\
& Estreptomicina & $0,08 \mathrm{~g}$ \\
& Água destilada q.s.p. & $100 \mathrm{~mL}$ \\
\hline \multirow{2}{*}{ Meio à base de leite } & Gicarbonato de Sódio & $0,15 \mathrm{~g}$ \\
desnatado (L) & Leite desnatado & $2,4 \mathrm{~g}$ \\
& Penicilina G Potássica & $0,056 \mathrm{~g}$ \\
& Estreptomicina & $0,07 \mathrm{~g}$ \\
& Água destilada q.s.p. & $100 \mathrm{~mL}$ \\
& & \\
\hline
\end{tabular}

bloco e o ejaculado após diluição, a unidade experimental. As variáveis estudadas foram motilidade e vigor nos períodos 0, 30, 60, 90 e 120 minutos.

A análise dos dados foi realizada empregando-se o programa SAS. ${ }^{16}$ Os índices obtidos de motilidade e vigor foram avaliados pelo PROC GLM do SAS (ANOVA) e quando verificado significância $(\mathrm{p}<0,05)$ procedeu-se o teste de Tukey.

\section{Resultados}

A motilidade progressiva diminuiu significativamente $(\mathrm{P}<0,05) \operatorname{logo}$ após a diluição em Ringer com lactato de sódio (R) em relação ao sêmen in natura. Contudo, não foram observadas diferenças $(\mathrm{P}>0,05)$ quando este parâmetro foi comparado entre as amostras de sêmen diluídas com os meios de citrato-gema (C) e à base de leite desnatado (L) (Tabela 2).

$\mathrm{O}$ vigor apresentou uma queda significativa $(\mathrm{P}<0,05)$ logo após a diluição em todos os diluidores testados quando confrontados com o sêmen in natura. Quando estabelecida uma comparação no tempo 0 , não se observou diferença $(\mathrm{P}>0,05)$ entre os três diluidores (Tabela 2).

Decorridos 30 minutos da incubação, o sêmen diluído em Ringer com lactato de sódio apresentou redução da motilidade espermática $(\mathrm{P}<0,05)$ mais pronunciada do que o sêmen diluído em citrato-gema e leite desnatado (Tabela 3). A diferença manteve-se até 120 minutos de incubação.

O vigor apresentou variações nos diferentes tratamentos no decorrer das avaliações (Tabela 4). No tempo 30, foi menor $(\mathrm{P}<0,05)$ na amostra de sêmen diluída em citrato-gema em relação ao que se adicionou o diluidor a base de leite, porém este não diferiu do diluído em Ringer com lactato de sódio. $\mathrm{Na}$ avaliação do tempo 60, os valores obtidos não foram diferentes $(\mathrm{P}>0,05)$ entre os três diluidores. Esta variável, no tempo 90, apresentou menor redução $(\mathrm{P}<0,05)$ no sêmen diluído em Ringer com lactato de sódio do que diluído em citrato-gema. Fato este, que não ocorreu nos espermatozóides diluídos em diluidor à base de leite desnatado, já que apresentaram valores de vigor semelhantes ( $>0$,05) aos outros diluidores testados. Com 120 minutos de incubação, todos os diluidores 
Tabela 2 - Médias \pm desvios padrão da motilidade e do vigor do sêmen de coelhos in natura e imediatamente após a diluição com Ringer com lactato de sódio, citrato-gema e meio à base de leite desnatado - Pirassununga, 2004

\begin{tabular}{lcc}
\hline Diluidor & Motilidade (\%) & Vigor (1 - 5) \\
\hline Sêmen in natura & $67,2 \pm 5,455^{\mathrm{a}}$ & $3,04 \pm 0,389^{\mathrm{a}}$ \\
Ringer com lactato de sódio & $64,5 \pm 8,763^{\mathrm{b}}$ & $2,85 \pm 0,527^{\mathrm{b}}$ \\
Citrato-gema & $67,0 \pm 5,345^{\mathrm{a}, \mathrm{b}}$ & $2,73 \pm 0,443^{\mathrm{b}}$ \\
Meio a base de leite & $65,9 \pm 5,316^{\mathrm{a}, \mathrm{b}}$ & $2,72 \pm 0,454^{\mathrm{b}}$ \\
\hline a,b Letras diferentes na mesma coluna indicam diferenças significativas $(\mathrm{P}<0,05)$.
\end{tabular}

Tabela 3 - Médias \pm desvios padrão da motilidade (\%) do sêmen de coelho diluído em Ringer com lactato de sódio, citrato-gema e meio à base de leite desnatado e incubados in vitro a $37^{\circ} \mathrm{C}$ por 120 minutos Pirassununga, 2004

\begin{tabular}{|c|c|c|c|c|c|}
\hline \multirow{2}{*}{ Tratamentos } & \multicolumn{5}{|c|}{ Tempo de Incubação } \\
\hline & $0 \mathrm{~min}$ & $30 \mathrm{~min}$ & $60 \mathrm{~min}$ & $90 \mathrm{~min}$ & $120 \mathrm{~min}$ \\
\hline $\mathbf{R}$ & $64,5 \pm 8,763^{\mathrm{a}}$ & $58,6 \pm 14,287^{a}$ & $56,3 \pm 14,977^{a}$ & $53,8 \pm 15,171^{a}$ & $50,3 \pm 16,946^{a}$ \\
\hline $\mathbf{C}$ & $67,0 \pm 5,345^{a}$ & $65,4 \pm 6,455^{b}$ & $63,1 \pm 9,252^{b}$ & $59,9 \pm 13,073^{b}$ & $57,5 \pm 14,006^{b}$ \\
\hline $\mathbf{L}$ & $65,9 \pm 5,316^{a}$ & $63,1 \pm 5,793^{b}$ & $61,1 \pm 6,085^{b}$ & $58,8 \pm 7,530^{b}$ & $55,8 \pm 8,041^{b}$ \\
\hline \multicolumn{6}{|c|}{$\begin{array}{l}\text { sb Letras diferentes na mesma coluna indicam diferenças significativas }(\mathrm{P}<0,05) ; \mathrm{R}=\text { Sêmen diluído em Ringer com lactato de sódio; } \mathrm{C}= \\
\text { Sêmen diluído em meio de citrato-gema; } \mathrm{L}=\text { Sêmen diluído em meio à base de leite desnatado }\end{array}$} \\
\hline \multirow{2}{*}{ Tratamentos } & \multicolumn{5}{|c|}{ Tempos } \\
\hline & $0 \mathrm{~min}$ & $30 \mathrm{~min}$ & $60 \mathrm{~min}$ & $90 \min$ & $120 \mathrm{~min}$ \\
\hline $\mathbf{R}$ & $2,85 \pm 0,527^{\mathrm{a}}$ & $2,56 \pm 0,531^{a, b}$ & $2,56 \pm 0,586^{a}$ & $2,53 \pm 0,584^{a}$ & $2,47 \pm 0,642^{\mathrm{a}}$ \\
\hline $\mathbf{C}$ & $2,73 \pm 0,443^{a}$ & $2,46 \pm 0,333^{a}$ & $2,42 \pm 0,369^{a}$ & $2,32 \pm 0,471^{b}$ & $2,28 \pm 0,564^{a}$ \\
\hline $\mathbf{L}$ & $2,72 \pm 0,454^{a}$ & $2,63 \pm 0,402^{b}$ & $2,49 \pm 0,385^{a}$ & $2,38 \pm 0,372^{a, b}$ & $2,47 \pm 0,642^{a}$ \\
\hline
\end{tabular}

${ }^{2 \cdot b}$ Letras diferentes na mesma coluna indicam diferenças significativas $(\mathrm{P}<0,05) ; \mathrm{R}=$ Sêmen diluído em Ringer com lactato de sódio; $\mathrm{C}=$ Sêmen diluído em Citrato-gema a 5\%; L = Sêmen diluído em meio à base de leite desnatado

apresentaram valores que não diferiam quando analisados estatisticamente $(\mathrm{P}>0,05)$.

\section{Discussão}

A escolha do Ringer com lactato de sódio e diluidor à base de citrato de sódio e gema de ovo, como diluidores para sêmen de coelhos, tiveram como base os resultados positivos obtidos anteriormente por diversos autores, quanto aos índices produtivos, taxa de prenhez e número de 
láparos nascidos vivos. ${ }^{7,11,12,17,18,19}$ Já o diluidor a base de leite desnatado tem mostrado sua capacidade na proteção dos espermatozóides da espécie eqüina ${ }^{20}$, além da sua eficiência, na conservação do sêmen de coelho, demonstrado pelo teste de exaustão a $37^{\circ} \mathrm{C}^{13}$.

Comparado a soluções salinas simples à inclusão da gema do ovo, em preparações usadas pra diluir o sêmen de coelho, resultou na melhora da motilidade dos espermatozóides. ${ }^{10}$ Resultado que coincide com os valores obtidos neste trabalho, no qual ao avaliarem a motilidade e o vigor, no decorrer do tempo pós-diluição, no tratamento que contém em sua composição a gema do ovo, notou-se que este apresentou melhor capacidade de preservar a motilidade do que a solução salina de Ringer com lactato de sódio.

Uzcatequi e Johnston ${ }^{21}$ não observaram diferenças na taxa de concepção e no número de láparos nascidos vivos, quando compararam inseminações realizadas com sêmen diluído em solução salina $(0,9 \%$ de $\mathrm{NaCl})$ ao adicionado ao diluidor à base de gema de ovo e citrato. Porém, estes autores citam que a solução salina só mantém a capacidade fertilizante do espermatozóide, caso este seja usado logo após a diluição. Ressalta-se que no presente experimento, os valores de motilidade encontrados imediatamente após a diluição (tempo 0) nas soluções Ringer com lactato de sódio e no citrato-gema não tiveram valores que diferissem $(\mathrm{P}>0,05)$ entre si.

Bicudo e Paschoal ${ }^{13}$ efetuaram a comparação entre o meio a base de leite e o Ringer com lactato de sódio, pelo teste de exaustão a $37{ }^{\circ} \mathrm{C}$, obtendo resultados que diferiram dos encontrados neste experimento. Os autores referidos encontraram valores mais baixos de motilidade espermática $(7,5 \%$ para Ringer com lactato de sódio vs $27,7 \%$ para meio à base de leite) após 120 minutos de realizada a diluição quando comparado aos valores obtidos na atual pesquisa, que em média, foram superiores a $50 \% \mathrm{em}$ ambos os tratamentos.
Perante a literatura consultada, não foram encontrados trabalhos enfocando o uso de diferentes diluidores sobre a característica vigor em espermatozóides, nem comparando o uso de diluidores a base de leite desnatado e a base de citrato e gema de ovo no sêmen de coelhos. Os resultados obtidos, não mostram diferenças na capacidade de manutenção do parâmetro vigor nos diferentes tratamentos testados.

Ressalta-se que os valores de motilidade semelhantes entre o sêmen diluído em citrato-gema e o adicionado ao meio à base de leite, até 120 minutos pós-diluição, sugere que ambos são viáveis para a diluição e conservação da qualidade do sêmen em um determinado período de tempo, sendo indistintos na escolha para o uso em inseminação artificial das coelhas.

$\mathrm{Na}$ pesquisa realizada, a motilidade espermática mostrou-se sempre inferior quando o sêmen foi diluído em Ringer com lactato de sódio do que em relação aos outros dois tratamentos, durante todas as avaliações, caracterizando menor eficiência deste produto para a conservação do sêmen de coelho. Contudo, é interessante ressaltar que o Ringer com Lactato de Sódio, como fora citado outrora, além da fácil obtenção foi comprovado como sendo bastante eficiente como extensor de volume para a IA, já que, Bicudo et al. ${ }^{12}$ e Andrade et al. ${ }^{11}$ ao usarem este meio para diluir o sêmen, obtiveram como resultado índices zootécnicos como taxa de prenhez e números de láparos nascidos vivos semelhantes à monta natural. Acrescentando que, a manutenção da motilidade, em torno de 50\% após 120 minutos de incubação, pode ser considerada como um bom índice, não se sugerindo o descarte do sêmen.

\section{Conclusões}

Com base nos resultados obtidos, dentro das condições de realização deste experimento, é possível concluir que os diluidores Ringer com lactato de sódio, citrato-gema $5 \%$ e diluidor à base de leite desnatado, tiveram a capacidade de preservar 
a motilidade e o vigor, proporcionando um meio que permite a manutenção da viabilidade espermática. Deste modo, possibilitando a realização da IA em até duas horas pós-diluição.

Os diluidores à base de citrato-gema a $5 \%$ e leite desnatado preservam melhor motilidade e vigor do que a solução de Ringer com lactato de sódio. Todavia, nota-se que o emprego da solução Ringer com lactato de sódio, apesar de ter valores de conservação da motilidade e vigor inferiores aos outros tratamentos, pode ser recomendado como um diluidor de primeira escolha, tendo em vista a sua facilidade de compra e de uso, e principalmente por manter motilidade e vigor das células espermáticas por um período de até duas horas acima dos padrões mínimos necessários para garantir boa taxa de fertilidade.

Com estes conhecimentos em mãos surge a possibilidade do uso desta biotecnologia de forma mais ampla, o que proporciona grandes benefícios para a cunicultura nacional, assim como ocorreu em outras espécies domésticas.

\section{Agradecimentos}

$$
\text { Ao CNPq. }
$$

\section{In vitro efficiency of three extenders for rabbit semen}

\section{Abstract}

The objective of this experiment was to compare in vitro efficiency of three rabbit semen extenders: sodium lactate Ringer solution, sodium citrate and yolk-egg medium, and skim milk-based medium, on maintenance of sperm vigour and motility. To that end 5 rabbits were utilized; ten semen collections were taken from each $(\mathrm{n}=50)$. The semen was collected by artificial vagina, and evaluated for volume, motility, vigour, and concentration. The semen was diluted $\left(20 \times 10^{6}\right.$ spermatozoa $/ \mathrm{mL}$ ) in pre-warmed micro tube at $37^{\circ} \mathrm{C}$ in the three extenders, and then it was incubated in water bath at $37^{\circ} \mathrm{C}$ during 120 minutes, performing evaluation every 30 minutes. Immediately after the dilution (time 0) the sperm motility was not different among extenders $(\mathrm{P}>0.05)$, however, decreased $(\mathrm{P}<0.05)$ in Ringer extender when compared to in natura semen. The vigor in time 0 decreased $(\mathrm{P}<0.05)$ in the three extenders. The sperm motility was better preserved $(\mathrm{P}<0.05)$ during the incubation from 30 to 120 minutes for the semen diluted in yolk egg-citrate and skim milk-based extenders than for the Ringer extender. The vigour preservation varied among the extenders during the in vitro incubation; however, it was similar among the three extenders after 120 minutes of incubation. Based on these results, it can be deduced that the tested extenders promote a medium that allows the maintenance of sperm viability so that artificial insemination can take place within two hours of post-dilution.

\section{Referências}

1 BERGONZONI, M. L.; ZAMBELLI, D.; SAMOGGIA, G. Influenza del diluitore, della temperatura e del período di conservazione del seme nella F. A. cunicola. Rivista di coniglicoltura, n. 4, p. 37-40, 1994.

2 CHINELLATO, A.; PANDOLFO, L.; DE BIASI, M.; MUNERATTI, M.; ZAMBONIM, M. R.; POLVERINO DE LAURETO, P.; FASOLATO, L. Fecondazione
Key words:

Rabbit.

Semen.

Extender.

Artificial insemination. artificiale: standardizzazione del metodo. Rivista di Coniglicoltura, n. 5, p. 49-51, 1991.

3 FACCHIN, E. Fecondazione artificiale, a che punto siamo. Rivista di Coniglicoltura, n. 10, p. 13-22, 1995.

4 KISS, J. Bons ventos. Globo Rural, v. 16, n. 185, p. 44-49, 2001.

5 MORRELL, J. M. Artificial insemination in rabbits. British Veterinary Journal, v.151, n. 3, p. 477-488, 1995. 
6 CASTELLINI, C.; LATTAIOLI, P. Effect of numbers of motile sperms inseminated on reproductive performance of rabbit does. Animal Reproduction Science, v. 57, p. 111-120, 1999.

7 BACHIN, D.; LENNA, G.; GIRALDO, M. GREGGIO, M.; NALETTO, G. Fecondazione artificiale, il futuro è già iniziato. Rivista di coniglicoltura, n. 12, p. 69-76, 1988.

8 CHEEKE, P. R. Rabbit production. Illinois: Interstate printers, 1987. p. 249-266.

9 HOLT, W. V. Basic aspects of frozen storage of semen. Animal Reproduction Science, v. 62, p. 3-22, 2000.

10 ADAMS, C. E. Induction of ovulation and A.I. techniques in the rabbit. The Veterinary Record, v. 91, n. 8, p. 194-197, 1972.

11 ANDRADE, A. F. C.; CELEGHINI, E. C. C.; YONEZAWA, L. A.; SPERS, A.; ARRUDA, R. P. Comparação entre inseminação artificial e monta natural em coelhos quanto a taxa de prenhez e número de láparos nascidos. Revista Brasileira de Reprodução Animal, v. 27, n. 3, p. 520-521, 2003.

12 BICUDO, S. D.; POLASTRE, R.; MOURA, A. S. A. M. T.; CARMELO, M. J. Inseminação artificial de coelhas submetidas a regime intensivo de reprodução. Revista Brasileira de Reprodução Animal, n. 1, p. 232-233, 1989. Suplemento 1.

13 BICUDO, S. D.; PASCHOAL, J. P. S. Emprego do ringer lactato ou do leite desnatado como diluidores do sêmen de coelho: teste de exaustão a $37^{\circ} \mathrm{C}$ e refrigeração. In: CONGRESSO BRASILEIRO DE REPRODUÇÃO ANIMAL, 9., 1991, Belo Horizonte. Anais... Belo Horizonte: Colégio Brasileiro de Reprodução Animal, 1991. v. 2, p. 458.
14 ANDRADE, A. F. C.; YONEZAWA, L. A.; CELEGHINI, E. C. C.; SPERS, A.; ARRUDA R. P. Um novo modelo de vagina artificial para coelhos. Revista Brasileira de Reprodução Animal, v. 26, n. 3, p. 201204, 2002.

15 KENNEY, R. M.; BERGMAN, R. V.; COOPER, W. L.; MORSE, G. W. Minimal contamination techniques for breeding mares: techniques and preliminary findings. Proceedings of the American Association Equine Practice, v. 21, p. 327-336, 1975.

16 SAS. STATISTICAL ANALISYS SYSTEM. SAS User's guide: statistical. Release 6.12. Cary: SAS, 1996.

17 BONANNO, A.; ALABISO, M.; ALICATA, M. L. Effeti del trattamento sincronizzante con PMSG su coniglie inseminate artificialmente. Rivista di Coniglicoltura, n. 11, p. 29-32, 1991.

18 CASTELLINI, C. Confronto tra fecondazione naturale e inseminazione artificiale com mestrui differenti nel coniglio. Rivista di Coniglicoltura, n. 2, p. 57-59, 1990.

19 CONSTANTINI, F. F. A. Nel coniglio, sistemi di conservazione dello sperma. Rivista di Coniglicoltura, n. 4, p. 14-18, 1989.

20 BATELLIER, F.; DUCHAMP, G.; VIDAMENT, M.; ARNAUD, G.; PALMER, E.; MAGISTRINI, M. Delayed insemination is successful with a new extender for storing fresh equine semen at 15 degrees centigrade under aerobic conditions. Theriogenology, v. 50, n. 2, p. 229236, 1998.

21 UZCATEQUI, M. E.; JOHNSTON, N. P. The use of ovulatory agents and semen diluents in the artificial insemination of New-Zealand and Rex rabbits. Journal Applied Rabbit Research, n. 11, p. 113-115, 1988. 